\title{
Editorial
}

\section{Dengue virus type 4 arrives in the state of Rio de Janeiro: a challenge for epidemiological surveillance and control}

Dengue virus type 4 (DENV-4) re-emerged in the state of Roraima (RR), a remote state in the extreme Northwest of Brazil, in July 2010 at the height of the rainy season. This serotype had not been detected in the country for almost 30 years, when DENV-4 and DENV-1 caused a limited outbreak in RR's capital city Boa Vista in 1981-1982 with 11,000 reported cases. The reinvasion of DENV-4 in July 2010 was not followed by an epidemic in RR; less than 20 cases of DENV-4 were confirmed in this state throughout the second half of 2010. The first cases, which were considered to have been the result of the virus spreading from RR, were not registered until January 2011. First, the virus reached two states of the Northern Region, Amazonas and Pará, in January. In March, the virus was subsequently detected in the Northeast Region, first in the state of Piauí and later in the states of Pernambuco, Bahia and Ceará, and in the Southeast Region, in the states of Rio de Janeiro (RJ) and São Paulo. The arrival of this serotype in RJ eight months after its resurgence in RR, almost 5,000 km away, has important and particular epidemiological significance. Furthermore, its early detection during the course of a DENV-1 epidemic reveals the efficiency of an epidemiological surveillance.

$\mathrm{RJ}$ is a hub for many tourist activities and is therefore connected to a large and busy roadway and railway system that allows access to the most populous part of the country. In addition, the local Aedes aegypti populations are highly susceptible to dengue virus and are generally found in high densities throughout most of the state's territory. As a result, the state is known as the most important point for the introduction and dissemination of the dengue virus in Brazil. Indeed, RJ was where the introduction and dispersion of the first cases of DENV-1 (1986; the previous invasion in 1981 was limited to Boa Vista), DENV-2 (1990) and DENV-3 (2000) took place. The recent introduction of DENV-4 in this state is of great concern because of its potential to spread to the rest of Brazil.

The first cases of DENV-4 detected in RJ occurred in the Cafubá neighbourhood, in the oceanic region of the municipality of Niterói $\left(22^{\circ} 52^{\prime} \mathrm{S} 43^{\circ} 06^{\prime} \mathrm{W} ; 487,327\right.$ inhabitants), which is located in the metropolitan region of RJ, only $15 \mathrm{~km}$ away from the capital city. The tourist sector is one of the most promising sectors, and the water supply reaches almost all of the households in the neighbourhoods' urban areas in Niterói. However, the sewage system does not coincide with the extension of the water supply networks. A considerable portion of the Niterói population is represented by the elderly, and the aging index is the highest in the state.

Curiously, the first two cases of DENV-4 in RJ were detected in two young sisters, 22 and 23 years of age, who first began to show symptoms on March 6, 2011 during a DENV-1 outbreak in the state. Both presented fever, cephalgia, arthralgia, myalgia, exanthema, vomiting and abdominal pain. One of the sisters was hospitalised as a result of the symptoms and hypotension. Her platelet counts were 132,000 and $72,000 \mathrm{~mm}^{3}$ with high levels of liver enzymes $(\mathrm{TGO}=106 \mathrm{U} / \mathrm{L}, \mathrm{TGP}=51 \mathrm{U} / \mathrm{L}$, gama GT $=303 \mathrm{U} / \mathrm{L}$, alkaline phosphatase $=104 \mathrm{U} / \mathrm{L}$, direct bilirubin $=0.2 \mathrm{mg} / \mathrm{dL}$, indirect bilirubin $=0.2 \mathrm{mg} / \mathrm{dL}$ and total bilirubin $=0.4 \mathrm{mg} / \mathrm{dL}$ ). Immediately after identifying the first case of DENV-4, the collection of blood samples for dengue virus research intensified in Niterói, particularly in the oceanic region, in order to monitor the dispersion of the virus. Five new cases were subsequently confirmed in Niterói: two cases in the Cafubá neighbourhood and three cases in the São Domingos neighbourhood (approximately $11 \mathrm{~km}$ from Cafubá), totalling seven cases of DENV-4 by the end of April, with referred abdominal pain in three of them. The three cases from São Domingos, a boy (14 years old), a girl (15) and her father (42), live on the same street. These cases have no epidemiological relation to those from Cafubá, confirming a quick viral spread in Niterói. Notably, São Domingos is adjacent to the main, busy campuses of the Fluminense Federal University, from which DENV-4 may rapidly spread through the state.

The virus was isolated in one of the cases by cell culture inoculation in the C6/36 cell line and the serotype was confirmed by indirect immunofluorescence using monoclonal antibodies. In addition, all cases were confirmed by polymerase chain reaction (PCR) in serum samples and by serology based on the presence of IgM antibodies. Preliminary data of partial sequencing suggests that the DENV-4 found in Niterói corresponds to the genotype presently circulating in other South American countries.

Since the 1990s, the oceanic region in Niterói has been going through an intense process of population growth and an increase in civil construction. This region has reported a significant number of dengue fever cases throughout the last 10 years and consistently contributes to viral circulation research by providing samples. Therefore, although the number of DENV-1 cases increased during the first trimester of 2011 in this region and in the municipality of Niterói as a whole, an efficient surveillance system enabled the early detection of the first cases of DENV-4. 
Since 1986, Niterói has established a surveillance system for dengue fever, including collecting blood for virus detection (culture inoculation and PCR) from fever cases until the fifth day after the onset of symptoms in Health Centres from the Sentinela network. The identification of DENV-4 confirmed the co-circulation of three serotypes, DENV-1, DENV-2 and DENV-4; DENV-3 has not been isolated since 2008.

Niterói's basic healthcare network consists of 54 units. Each unit has at least one surveillance professional, which allows surveillance and assistance to work well together, making information flow faster. The experience gained from past epidemics is presented in meetings, in capacity-building courses for professionals and in informational materials. The epidemiological surveillance office publishes a bulletin (Informe dengue) that is released every week during the warning phase and when the epidemic has set. All Health Units offer blood collection services and other materials for laboratory exams, and the Municipal Health Foundation has its own network of laboratories made up of three laboratories that render services for the basic healthcare network and four laboratories for the hospital network.

The dengue fever virological surveillance protocol currently in use recommends collecting blood samples for serology tests in fever cases after the seventh day of the disease (children, expecting mothers, elderly, people with comorbidities, severe cases), in addition to investigating deaths. Patients who receive medical care until the fifth day of the disease (feverish period) are eligible to have samples collected for viral isolation.

The municipality of Niterói has stood out in the epidemiology of dengue fever in Brazil, with the early description of dengue fever's clinical picture in 1923 by Antonio Pedro. Furthermore, it was in this municipality that DENV-2 was first isolated in April 1990. Two months later, Niterói was where the first case of dengue haemorrhagic fever in Brazil was registered based on criteria from the World Health Organization. Curiously, the first isolation of DENV-2 (1990) and DENV-4 (2011) in RJ occurred during an outbreak of DENV-1 (Supplementary data).

Historically, the arrival of a new type of dengue fever in RJ has shown to be explosive during the rainy seasons after the detection of the first cases, following a rapid dissemination into the large metropolitan area and to other states, as described by the virological research and surveillance conducted by the late Dr Hermann Schatzmayr's team during the past 25 years. The arrival of DENV-4 in RJ represents once more another great challenge for epidemiological surveillance, control and healthcare in Brazil.

The situation of hyperendemicity in the state (several co-circulating serotypes) of a virus of renowned pathogenicity in RJ, as well as the possibility of sequential infections, has led to the appearance of severe forms of the disease in the state, even in children. Therefore, providing clinical management, organizing healthcare and maintaining an efficient surveillance system are of the utmost importance in reducing dengue mortality.

Rita MR Nogueira Laboratório de Flavivírus, Instituto Oswaldo Cruz-Fiocruz, Rio de Janeiro, RJ, Brasil 


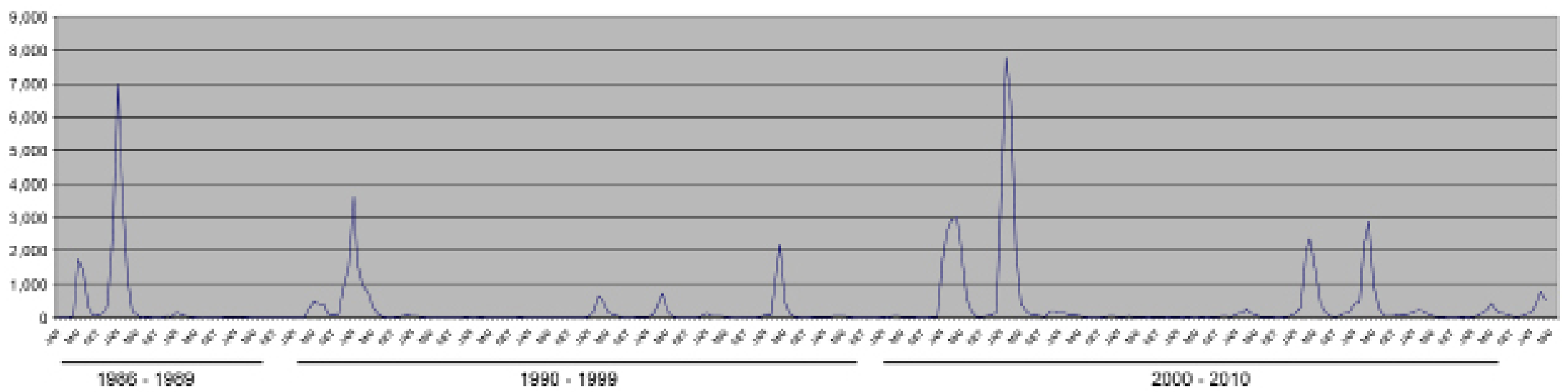

Number of dengue cases in Niterói, state of Rio de Janeiro, Brazil. Source: Coordenação de Vigilância em Saúde/Fundação Municipal de Saúde de Niterói (data obtained in April 26 2011)

Number of dengue cases in Niterói, state of Rio de Janeiro, Brazil

\begin{tabular}{|c|c|c|c|c|c|c|c|c|c|c|c|c|c|c|c|c|c|c|c|c|c|c|c|c|c|c|}
\hline & 1986 & 1987 & 1988 & 1989 & 1990 & 1991 & 1992 & 1993 & 1994 & 1995 & 1996 & 1997 & 1998 & 1999 & 2000 & 2001 & 2002 & 2003 & 2004 & 2005 & 2006 & 2007 & 2008 & 2009 & 2010 & 2011 \\
\hline JAN & 0 & 6,980 & 141 & 25 & 3 & 3,636 & 47 & 5 & 1 & 21 & 33 & 116 & 83 & 24 & 16 & 1,721 & 3,330 & 126 & 37 & 30 & 107 & 132 & 497 & 177 & 13 & 162 \\
\hline FEB & 0 & 3,562 & 85 & 31 & 4 & 1,592 & 47 & 11 & 3 & 186 & 68 & 56 & 80 & 30 & 11 & 2,553 & 7,778 & 122 & 24 & 11 & 152 & 307 & 565 & 219 & 46 & 375 \\
\hline MAR & 0 & 1,117 & 41 & 17 & 5 & 952 & 21 & 34 & 8 & 660 & 384 & 52 & 1,001 & 61 & 38 & 2,944 & 6,432 & 80 & 38 & 19 & 264 & 1,916 & 2,636 & 199 & 118 & 778 \\
\hline APR & 42 & 213 & 21 & 3 & 283 & 760 & 4 & 3 & 1 & 421 & 740 & 48 & 2,181 & 46 & 42 & 2,997 & 2,006 & 69 & 28 & 6 & 137 & 2,363 & 3,407 & 103 & 230 & 493 \\
\hline MAY & 1,745 & 65 & 4 & 3 & 466 & 326 & 4 & 0 & 7 & 184 & 235 & 23 & 459 & 46 & 35 & 1,861 & 404 & 42 & 19 & 3 & 78 & 1,476 & 1,125 & 56 & 433 & \\
\hline JUN & 1,390 & 12 & 0 & 5 & 405 & 92 & 3 & 0 & 2 & 67 & 58 & 10 & 89 & 19 & 18 & 549 & 229 & 13 & 13 & 14 & 34 & 459 & 272 & 28 & 196 & \\
\hline JUL & 315 & 16 & 1 & 1 & 400 & 19 & 2 & 0 & 1 & 41 & 9 & 7 & 32 & 12 & 15 & 211 & 96 & 6 & 12 & 10 & 22 & 174 & 59 & 19 & 116 & \\
\hline AUG & 60 & 5 & 2 & 0 & 91 & 12 & 1 & 4 & 1 & 11 & 5 & 4 & 12 & 6 & $3 t$ & 23 & 72 & 6 & 27 & 24 & 14 & 45 & 39 & 9 & 111 & \\
\hline SEP & 76 & 4 & 1 & 0 & 76 & 7 & 3 & 3 & 1 & 5 & 8 & 4 & 10 & 10 & 7 & 19 & 42 & 6 & 36 & 35 & 8 & 35 & 42 & 5 & 44 & \\
\hline OCT & 123 & 6 & 11 & 4 & 96 & 25 & 3 & 6 & 6 & 10 & 8 & 6 & 4 & 2 & 7 & 38 & 37 & 15 & 33 & 41 & 9 & 38 & 108 & 5 & 29 & \\
\hline $\mathrm{NOV}$ & 348 & 38 & 21 & 2 & 881 & 43 & 3 & 14 & 1 & 31 & 12 & 12 & 20 & 4 & 19 & 66 & 190 & 46 & 12 & 45 & 22 & 120 & 80 & 16 & 50 & \\
\hline DEC & 2,472 & 25 & 34 & 3 & 1,531 & 86 & 29 & 10 & 2 & 22 & 48 & 26 & 16 & 8 & 18 & 154 & 189 & 41 & 32 & 37 & 41 & 165 & 94 & 8 & 136 & \\
\hline TOTAL & 6,571 & 1,2043 & 362 & 94 & 4,241 & 7,550 & 167 & 90 & 34 & 1,659 & 1,608 & 364 & 3,987 & 268 & 229 & 13,136 & 20,805 & 572 & 311 & 275 & 888 & 7,230 & 8,924 & 844 & 1,522 & 1,808 \\
\hline
\end{tabular}

Source: Coordenação de Vigilância em Saúde/Fundação Municipal de Saúde de Niterói (data obtained in April 26 2011).

Some historical remarks on dengue virus circulation

in the municipality of Niterói, state of Rio de Janeiro, 1986-2011

\begin{tabular}{|c|c|}
\hline 1923 & First clinical description of dengue fever \\
\hline 1986/1987 & Re-emergence of dengue: isolation of dengue fever type 1 (DENV-1) \\
\hline 1990/1991 & Isolation of DENV-2 (for the 1st time in Brazil) \\
\hline 1995/1996/1998 & Continuous isolations of DENV-2 \\
\hline $2001 / 2002$ & $\begin{array}{l}\text { Severe dengue outbreak: } 33,941 \text { reported cases } \\
210 \text { cases of dengue hemorrhagic fever (DHF) } \\
199 \text { hospitalizations } \\
\text { six deaths due to DHF in } 2002 \text { (for the 1st time in Brazil) } \\
\text { Isolation of DENV-1 (2001) and DENV-3 (2002) }\end{array}$ \\
\hline $2007 / 2008$ & $\begin{array}{l}\text { Dengue epidemics: } 16,054 \text { reported dengue cases } \\
\text { Re-emergence of DENV-2 (2008) } \\
284 \text { cases of dengue with complications and DHF; two deaths }\end{array}$ \\
\hline $2009 / 2010$ & $\begin{array}{l}\text { Non epidemic period } \\
\text { Isolation of DENV-2 } \\
\text { Gradual augmentation reports of dengue cases (high endemicity) }\end{array}$ \\
\hline 2011 & Isolation of DENV-1, DENV-2 and DENV-4 \\
\hline
\end{tabular}

Source: Coordenação de Vigilância em Saúde/Fundação Municipal de Saúde de Niterói (data obtained in April 26 2011). 